\title{
Antibodies to actin in autoimmune haemolytic anaemia
}

\author{
Kathrin M Felder ${ }^{1}$, Katharina Hoelzle ${ }^{1}$, Karl Heinritzi ${ }^{2}$, Mathias Ritzmann ${ }^{3}$, Ludwig E Hoelzle ${ }^{1 *}$
}

\begin{abstract}
Background: In autoimmune haemolytic anaemia (AlHA), autoreactive antibodies directed against red blood cells are up-regulated, leading to erythrocyte death. Mycoplasma suis infections in pigs induce AlHA of both the warm and cold types. The aim of this study was to identify the target autoantigens of warm autoreactive IgG antibodies. Sera from experimentally $M$. suis-infected pigs were screened for autoreactivity.

Results: Actin-reactive antibodies were found in the sera of $95 \%$ of all animals tested. The reactivity was speciesspecific, i.e. reactivity with porcine actin was significantly higher than with rabbit actin. Sera of animals previously immunised with the M. suis adhesion protein MSG1 showed reactivity with actin prior to infection with M. suis indicating that molecular mimicry is involved in the specific autoreactive mechanism. A potentially cross-reactive epitope was detected.
\end{abstract}

Conclusions: This is the first report of autoreactive anti-actin antibodies involved in the pathogenesis of autoimmune haemolytic anaemia.

\section{Background}

Haemotrophic mycoplasma infections are found in a wide range of domestic and wild animals causing acute haemolytic anaemia or chronic anaemia with immune suppression [1,2]. Generally, they show distinct host specificity. However, there are reports of humans infected with haemotrophic mycoplasmas, i.e. Mycoplasma haemofelis and Mycoplasma suis [3,4]. M. suis causes infectious anaemia in pigs (IAP) [1].

Two forms of autoimmune haemolytic anaemia (AIHA) have been described: warm AIHA, which is characterised by autoreactive IgG antibodies binding their target epitope at body temperature [5], and cold AIHA. In cold AIHA, complement-activating autoreactive IgM antibodies bind their antigen, usually the $1 / \mathrm{i}$ epitope on red blood cells, below body temperature and are strongly agglutinating at $4^{\circ} \mathrm{C}[6,7]$. Both types of antibodies occur in 7\% of human AIHA cases; this is referred to as mixed AIHA. Sokol and co-workers report only one case of secondary mixed AIHA due to Mycoplasma infection, i.e. M. pneumoniae [8].

\footnotetext{
* Correspondence: Ihoelzle@vetbakt.uzh.ch

${ }^{1}$ Institute of Veterinary Bacteriology, Vetsuisse Faculty, University of Zurich, Switzerland
}

Mixed AIHA has been found in M. suis-induced IAP. Cold IgM agglutinins targeting glycoproteins on the red blood cells were observed $[9,10]$. These cold agglutinins occur about four weeks after experimental infection with $M$. suis. During the acute stage of IAP (clinical attack), acute anaemia, hypoglycaemia and icteroanaemia accompanied by high mortality occur. It is assumed that the severe anaemia observed in pigs suffering from acute clinical signs is due to a combination of direct damage to the red blood cells by $M$. suis attachment and invasion, and upregulation of cold and warm autoreactive antibodies directed against red blood cell components. In previous studies, these autoreactive warm IgG antibodies have been shown to overwhelm the specific immune response to $M$. suis [11].

In this study, sera from experimentally M. suisinfected pigs were screened for the presence of warm IgG antibodies by testing their reactivity with blood preparations from healthy pigs. The detection and characterisation of the host proteins acting as autoantigens was a main issue. Earlier studies, i.e. serological proteome analysis [12], yielded some evidence that actin is a target protein for the autoreactive antibodies detected during the acute state of the disease. This was observed in both one- and two-dimensional immunoblots. 
Therefore, the sera tested in this study were additionally screened for reactivity with porcine muscular $(\alpha-)$ and cytoskeletal ( $\beta-)$ actin.

\section{Results \\ Study design and screening of porcine sera for autoreactive antibodies}

A collection of sera obtained from experimentally M. suis-infected pigs was available from earlier studies $[11,13]$. The sera were divided into two groups according to their history. Group 1 (36 sera) were derived from pigs $(\mathrm{n}=13)$ that had been vaccinated with MSG1 prior to splenectomy and challenge with $M$. suis. In view of the known major occurrence of autoreactive IgG antibodies during clinical attacks [11], the sera used for the present study were drawn at following time points: I, 21 days post-immunisation and 14 days prior to M. suis infection; II, during the first clinical attack (on average 10 days after infection); III, 14 days apart; IV, during the second clinical attack (on average 6 weeks post-infection). Owing to animal deaths, fewer sera were analysed at time points II $(\mathrm{n}=11)$, III $(\mathrm{n}=6)$ and IV $(\mathrm{n}=6)$. Group 2 (40 sera) originated from 10 pigs that had not been immunised prior to splenectomy and $M$. suis infection. For this study, sera taken prior to infection (I), during the first clinical attack (II), between the first and the second clinical attacks (III) and during the second clinical attack (IV) were considered. In total, 76 sera (36 of group 1 and 40 of group 2) were screened by ELISA for reactivity with IgG-depleted antigen preparations from healthy pigs (neg $\mathrm{Ag}$ ). OD values were normalised to the negative control. The cut-off value was determined to be 0.226 (three times the standard deviation of negative controls).

Figure 1A gives an overview of the screening results. At time point I, prior to splenectomy and infection, $84.6 \%$ of group 1 sera (immunised with MSG1) and no group 2 sera showed autoreactivity. There was a shift in antibody response during the first clinical attack. The reactivity of group 1 sera decreased to $45.5 \%$, and $60.0 \%$ of group 2 sera reacted with neg Ag. Between two clinical attacks, $83.3 \%$ of the sera from immunised pigs showed autoreactivity, and antibodies to neg Ag were completely down-regulated in the group 2 sera. During the second clinical attack, $100.0 \%$ of sera in group 1 and $80.0 \%$ of those in group 2 were autoreactive. Reactivities differed significantly between the groups at every time point. The $P$ values were $\leq 0.006,0.010,0.004$ and 0.043 for I, II, III and IV, respectively. In only one of the 23 animals were there no autoreactive antibodies in sera taken at any time point. Figure 1B shows the occurrence of autoreactive antibodies over the time course of infection in each group. The vaccinated animals representing group 1 had already produced autoreactive antibodies after immunisation with MSG1 and prior to infection and splenectomy, and these antibodies remained at a similar level for time points II, III and IV. The animals of group 2 showed no autoreactive antibodies before infection, up-regulated antibodies to neg Ag at time point II ( $1^{\text {st }}$ clinical attack), down-regulated antibodies below the cut-off value between clinical attacks, and production of autoreactive antibodies again during the $2^{\text {nd }}$ clinical attack (IV).

\section{Reactivity with porcine $\alpha$-actin}

In order to identify the autoantigens, the autoreactive sera $(n=36)$ were further tested with IgG-depleted erythrocyte lysates (ECL) and with porcine $\alpha$-actin. Earlier studies suggested actin as a potential target of warm autoreactive antibodies (one- and two dimensional immunoblots) [12]. In 32 sera (88.9\%), antibodies reactive with both preparations were detected. Linear regression for neg Ag with ECL and $\alpha$-actin revealed $R^{2}$ values of 0.732 and 0.744 , respectively (Fig. 2).

The actin reactivity was shown to be species-specific, i.e. a significantly lower reactivity was observed with rabbit $\alpha$-actin than with porcine $\alpha$-actin. The mean OD values at $\lambda=405 \mathrm{~nm}$ were $0.408 \pm 0.136$ and $0.793 \pm$ 0.185 for rabbit and porcine $\alpha$-actin, respectively.

\section{Reactivity with porcine $\beta$-actin}

The erythrocyte contains cytoskeletal actin ( $\beta$-actin). This actin form is highly homologous but not identical to muscle actin ( $\alpha$-actin). Since $\beta$-actin was not commercially available, the gene was synthetically produced and adapted to E. coli codon usage. The protein was expressed, purified and used for the experiments (Fig. 3). Sera reactive with $\alpha$-actin were tested further with $\beta$-actin. They were all positive for both actin forms. Group 1, the MSG1-immunised animals, showed a strikingly stronger reactivity with $\beta$-actin than with $\alpha$-actin $(P \leq 0.0002)$. No significant difference was seen in the group 2 animal sera $(P \leq 0.2034)$.

\section{Isotypes of autoreactive antibodies to actin}

In warm autoimmune haemolytic anaemia, $\operatorname{IgG}_{1}$ and $\mathrm{IgG}_{3}$ antibodies predominate [14]. These antibodies are recognised preferentially by macrophages. To gain insight into the autoreactive mechanisms occurring during an $M$. suis infection, secondary antibodies to porcine $I_{g} G_{1}$ and $I g G_{2}$ were used to evaluate the subtypes of actin-reactive antibodies in group 1 . No secondary antibodies to porcine $\operatorname{IgG}_{3}$ were available. The $\operatorname{IgG}_{1} / \operatorname{IgG}_{2}$ and $I g G_{2} / I_{g G}$ ratios were calculated to be $1.280 \pm$ 1.796 and $2.727 \pm 1.925$, respectively $(P \leq 0.018)$.

\section{Search for shared epitopes between MSG1 and actin}

Reactivity with actin was observed in MSG1-vaccinated immunocompetent animals prior to splenectomy and 


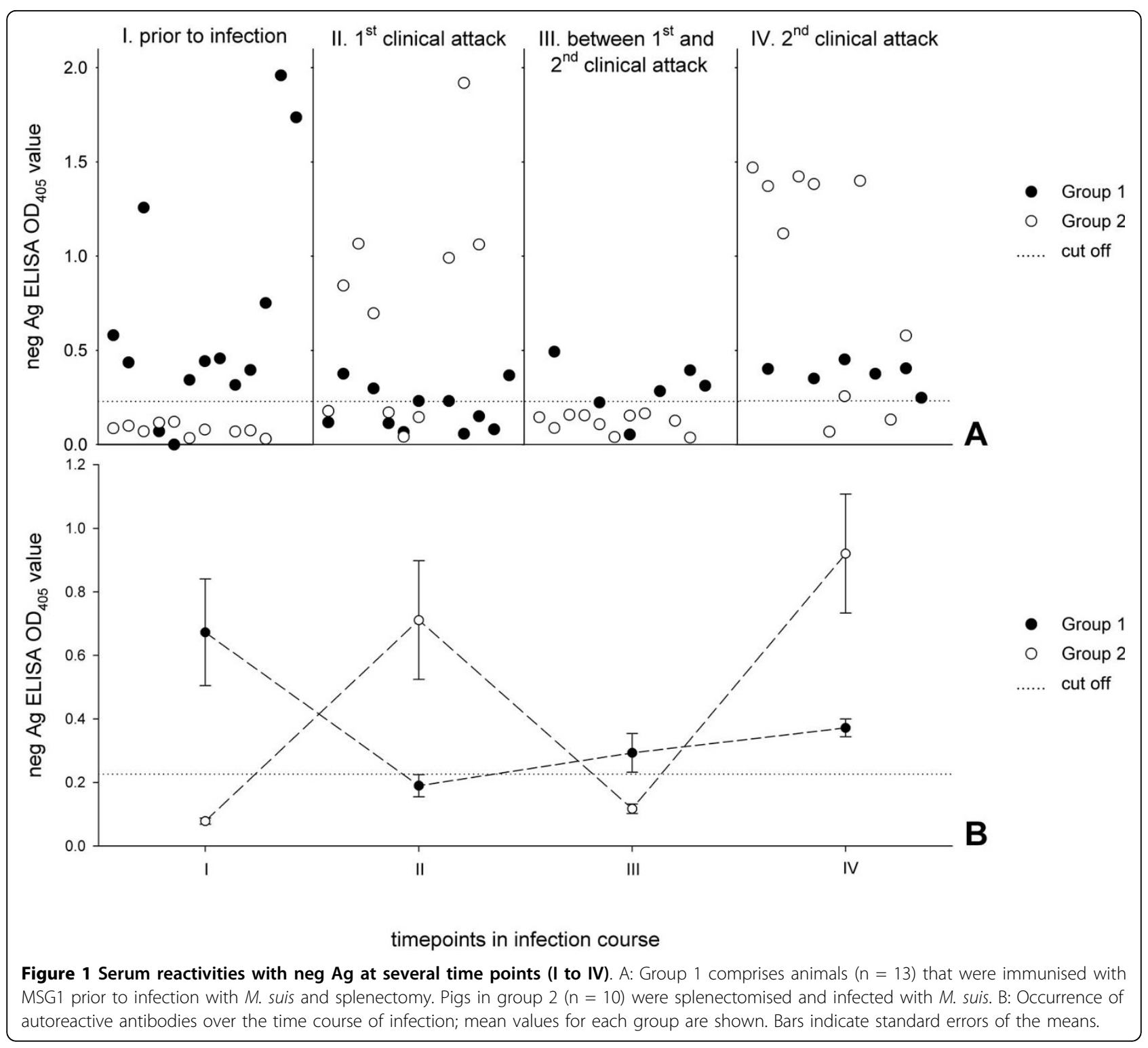

M. suis infection. To support the hypothesis of molecular mimicry, we evaluated the reactivity of a rabbit serum targeting recombinant MSG1 with actin and vice versa, i.e. the reactivity of a serum recognising porcine actin with MSG1. Cross-reactivity was observed (Fig. 4). Therefore, the protein sequences of porcine actin and MSG1 were used as input to an epitope finder program. The program allows epitopes potentially presented by SLA molecules to B-cells to be identified with high probability. SLA $-2 \% 0201$ would present the peptide LTLKYPIEH derived from actin and the peptide RTLKYYISL derived from MSG1. Both peptides are nine amino acids in length and share a sequence identity of $55 \%$. This peptide is identical in both actin forms.

\section{Discussion}

In $M$. suis infections, autoreactive antibodies are of central importance for inducing anaemia $[9,10]$. Recently we showed that warm autoreactive antibodies of the isotype IgG are up-regulated during the acute phase of experimentally-induced M. suis infection [11]. Twodimensional immunoblot analysis revealed that actin is potentially recognised by these antibodies [11,12]. Actin is known to play a role in autoimmune hepatitis type 1 (AIH-1), where F-actin-reactive antibodies are characteristic [15]. To our knowledge, this is the first report and evidence for autoreactive antibodies directed against actin in warm AIHA. In particular, characterising these autoantigenic structures is an essential basis for 


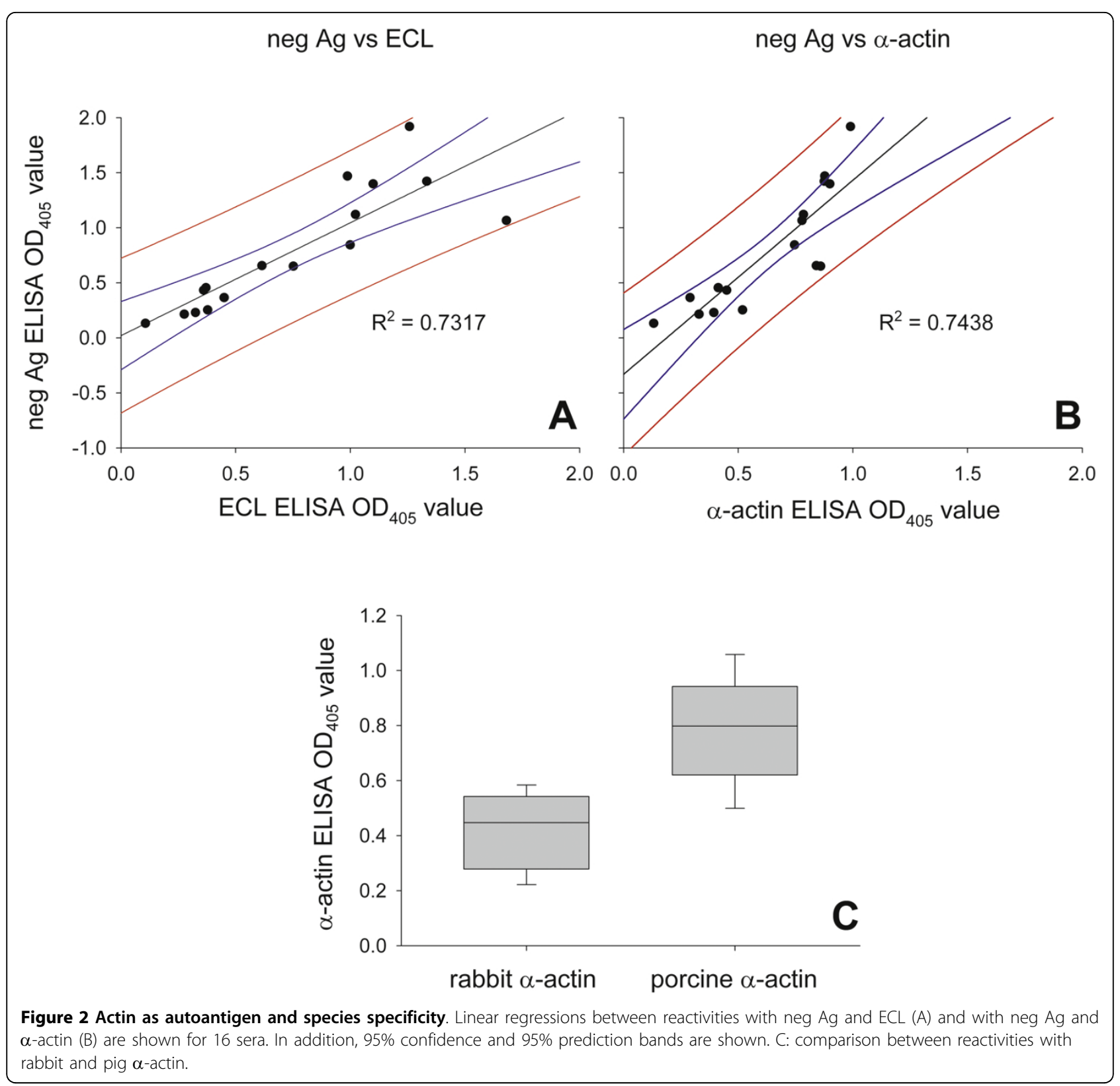

understanding the pathogenesis of warm AIHA, whether associated with $M$. suis infections or in general. Typically, autoreactive antibody production is induced by a misguided up-regulation of naturally-occurring B-cells specific for self-antigens, the occurrence of altered selfantigens, the appearance of previously cryptic antigens, and loss of tolerance to self-antigens due to molecular mimicry.

Antibodies recognising cytoskeletal components such as actin, spectrin or band 3 are physiological components of tissue homeostasis and B-cell clones specific for actin patrol continually [16]. A misregulation due to $M$. suis infection could lead to excessive thymus-independent proliferation of these B-cell clones [10]. Other Mycoplasma species, e.g. M. pneumoniae, are polyclonal B-cell activators of mouse splenocytes [17]. Further, Zachary and Smith have described such misdirected immune responses in M. suis infections [10]. Interestingly, actintargeting IgG antibodies are up-regulated during the first clinical attack when $M$. suis is present in large numbers. This provides evidence for the hypothesis of a direct mitogenic stimulus derived from $M$. suis leading to nonspecific B-cell stimulation.

A further explanation for the development of autoreactive antibodies is that damage to red blood cells makes hidden cytoskeletal proteins accessible to 


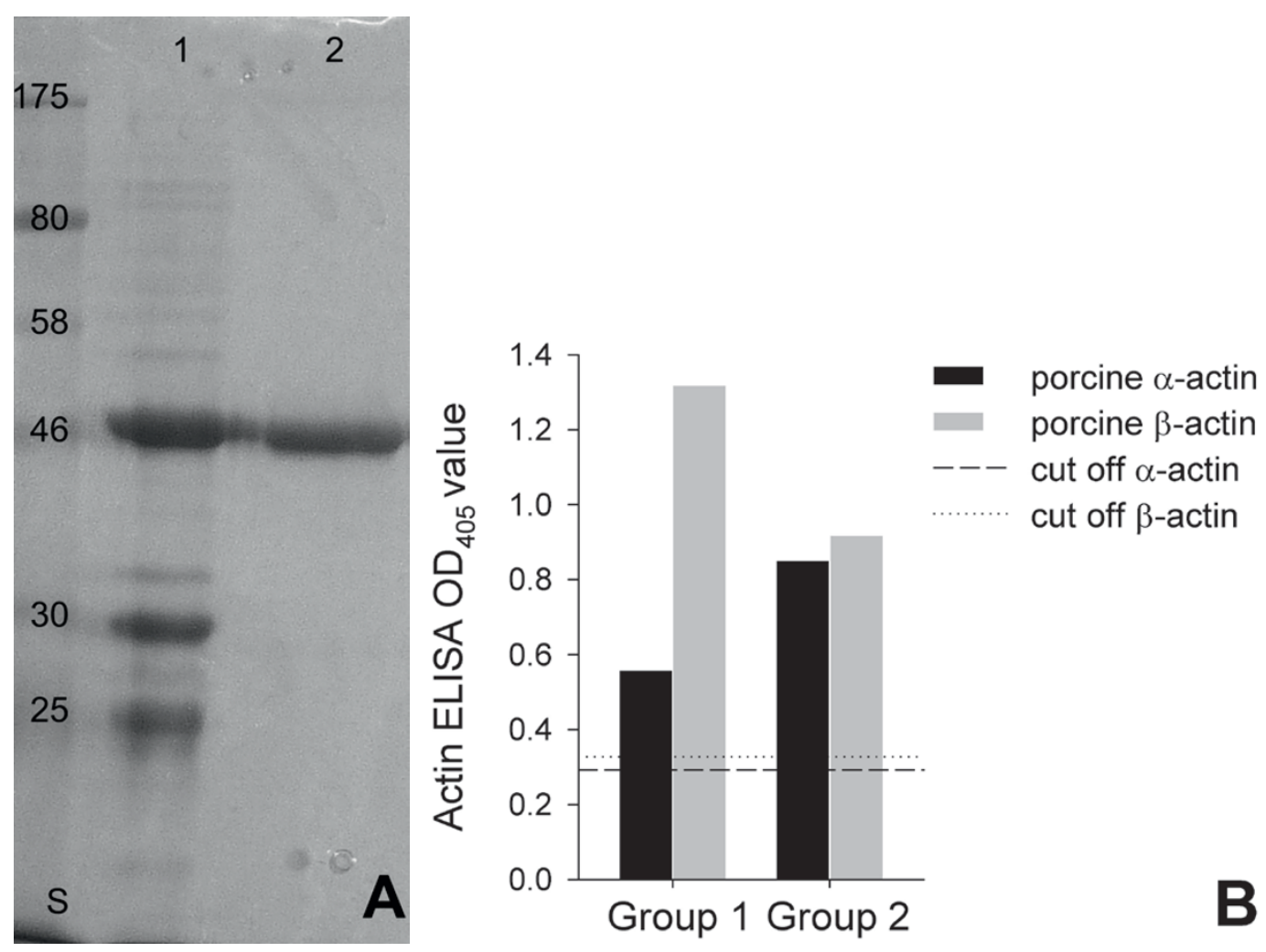

Figure 3 Purification of porcine $\beta$-actin and comparison of serum reactivities with $\boldsymbol{\alpha}$-actin and $\boldsymbol{\beta}$-actin. A: Coomassie-stained polyacrylamide gel showing purification of recombinant porcine $\beta$-actin. Nickel affinity chromatogryphy (1) and electro-elution (2) are shown. Protein mass standards in kDa are indicated in column S; B: Comparison of reactivity with $\alpha$-actin and $\beta$-actin. Group 1, i.e. immunised animals, showed significantly stronger reaction with cytoskeletal actin $(P \leq 0.0002)$. No difference between $\alpha$-and $\beta$-actin reactivities was observed in group 2 sera $(P \leq 0.2034)$.

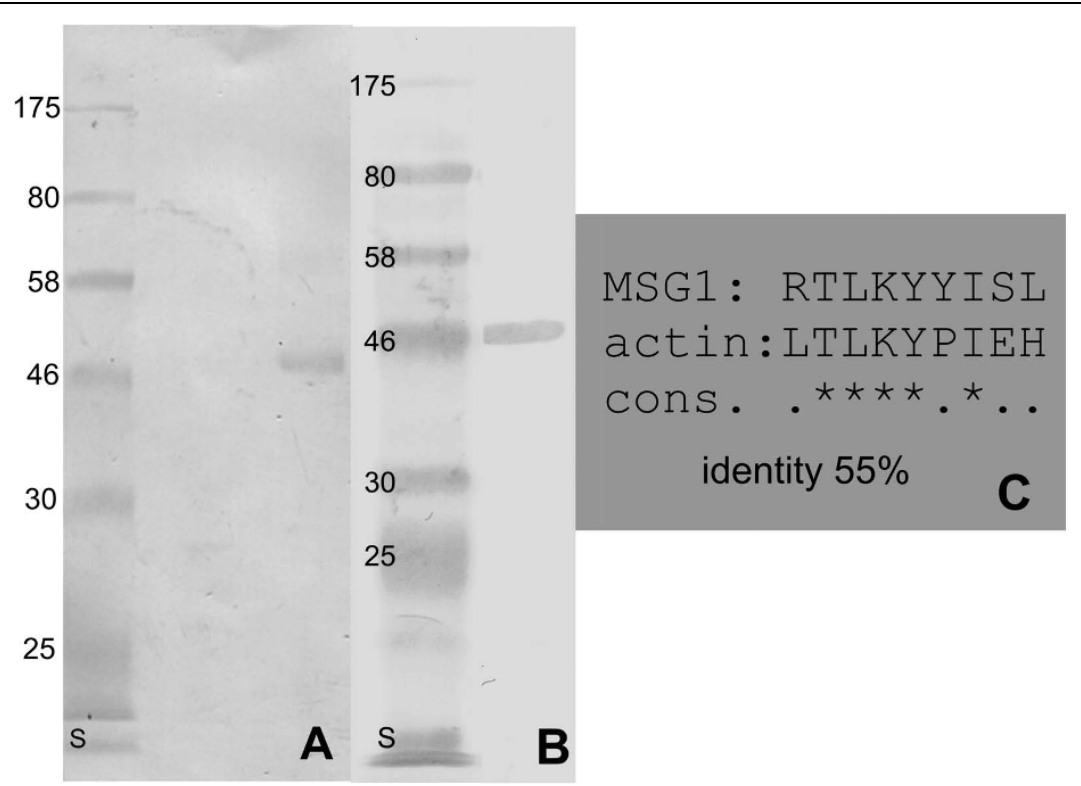

Figure 4 Cross-reactivity of rabbit hyperimmune sera between actin and MSG1. A: Westernblot reactivity of MSG1 with a rabbit serum specific for porcine $\alpha$-actin; B: Porcine actin is detected by a rabbit serum specific for MSG1, column S in A and B indicates protein masses in kDa; C: potential cross-reactive nonapeptides that would be presented by SLA*002 molecule. They share an identity of 55\%. 
circulating antibodies, whereupon they are interpreted as non-self and elicit an immune response. It has been hypothesised that autoimmune epitopes on red blood cells may be result from contact with proteolytic enzymes [18]. Electron microscopic studies have revealed a close association between $M$. suis cells and erythrocytes and striking membrane deformations on the host cells $[19,20]$. Considering loads of up to 4000 $M$. suis cells per erythrocyte, it is quite evident that the cytoskeleton is modified by attachment and invasion.

The last explanation for the induction of autoreactive antibodies directed against host actin in M. suis infections is molecular mimicry. In this process, a peptide derived from a pathogen and presented by MHC II must be capable of activating a self-reactive helper $\mathrm{T}$ cell and, therefore, stimulating specific antibodyproducing B-cells.

Remarkably, in sera obtained from pigs immunised with MSG1, autoantibodies against actin were already detectable after immunisation but prior to splenectomy and challenge with M. suis. These findings strongly support the hypothesis that MSG1 is involved in inducing autoimmunity by molecular mimicry. Evidence for cross-reactivity between MSG1 and actin was found by western blotting using hyperimmune rabbit sera. To test whether actin and M. suis actually share potentially cross-reactive epitopes, the NetMHCpan algorithm was used and gave a positive result [21]. Porcine SLA2*0201 (swine lymphocyte antigen) would strongly bind and therefore present the deduced peptides LTLKYPIEH (from porcine actin) and RTLKYYISL (from MSG1) to the same circulating T-cells. The identity between these peptides was calculated to be $55 \%$, which is enough for cross-reactivity. Degeneracy in both the TCR and MHC peptide-binding motifs reduces the sequence-specific requirement to only a few crucial residues [22-24]. It remains to be elucidated by systematic in vitro stimulation assays whether these peptides actually play a role in the pathogenesis of AIHA due to $M$. suis infection.

In RBCs and many other cell types $\beta$-actin is part of the cytoskeleton; $\alpha$-actin predominates only in muscle cells. If the strongly $\beta$-actin-binding antibodies identified encounter their target ubiquitously, RBCs would not be the only cells affected. There is evidence that lymphocytes and epithelial cells are harmed during M. suis infection (unpublished observations). Cytoskeletal proteins in cells with intact plasma membranes are cryptic for antibodies. However, if the cell is injured by interaction with $M$. suis, $\beta$-actin would become accessible. Antibodies recognising this actin opsonise the red blood cells which leads to their removal from the circulatory system and therefore to anaemia. Notably, clinical signs expected by autoreactive processes targeting muscle actin ( $\alpha$-actin) were observed.

In autoimmune diseases including AIHA, misdirected $\mathrm{IgG}_{1}$ and $\mathrm{IgG}_{3}$ antibodies play an important role because they bind efficiently to FcReceptorIII (FcRIII) molecules on macrophages $[14,25]$. Interestingly, the antibodies that predominantly recognised actin were of the subtype $\mathrm{IgG}_{2}$ rather than $\operatorname{IgG}_{1}$. However, a definitive subtype assignment was not feasible owing to high standard deviations. Immune complexes with the $\mathrm{IgG}_{2}$ subtype activate the alternative complement cascade pathway and are important in defence against encapsulated bacteria $[26,27]$. However, the consequences of autoreactive $\mathrm{IgG}_{2}$ up-regulation during $M$. suis infection remain unclear. Unfortunately, no statement about the presence of $\mathrm{IgG}_{3}$ antibodies and their role in $M$. suis infection can be given since secondary antibodies for this porcine subtype were not available.

\section{Conclusions}

Overall, we record that autoreactive IgG antibodies upregulated during infectious porcine anaemia induced by M. suis recognise actin. These autoreactive antibodies are obviously involved in the pathogenesis of the severe anaemia observed during the infection. An interplay of several mechanisms is assumed, i.e. a blastogenic response due to unspecific lymphocyte proliferation, direct damage to red blood cells and molecular mimicry. To our knowledge, this is the first report of autoreactive actin antibodies in relation to AIHA. In our opinion, the experimentally $M$. suis-infected splenectomised pig represents a useful model for investigating AIHA because of the controllable inducible symptoms.

\section{Methods}

\section{Preparation of screening antigens}

To screen for autoreactive antibodies, blood from healthy pigs was prepared as previously described for $M$. suis antigen preparations $[28,29]$ with slight modifications. Briefly, blood cells from $M$. suis-negative pigs were sedimented by centrifugation at $300 \times$ g for 15 min at room temperature (RT). Plasma and buffy coat were discarded. The erythrocytes were suspended in phosphate-buffered saline $(1 \times$ PBS; Biochrom Ag, Berlin, Germany) containing 0.15\% Tween 20 and 3\% EDTA and incubated for $20 \mathrm{~min}$ at RT with shaking. Debris and erythrocytes were removed by low-speed centrifugation $(500 \times \mathrm{g}, 20 \mathrm{~min}, \mathrm{RT})$. The supernatant was centrifuged at $25,000 \times \mathrm{g}$ for $120 \mathrm{~min}$ at $4^{\circ} \mathrm{C}$. The resulting pellet was resuspended $(1 \mathrm{mg} / \mathrm{ml})$ in sterile $1 \times$ PBS. The preparation was depleted of IgG and albumin using 
a ProteoExtract ${ }^{\circ}$ Albumin/IgG Removal Kit, Maxi (Calbiochem, Merck, Geneva, Switzerland) according to the manufacturer's recommendations and stored in $100 \mu \mathrm{l}$ aliquots at $-80^{\circ} \mathrm{C}$ until further use. The preparations were referred to as negative antigen (neg Ag).

Sera reactive with neg Ag were further tested with IgG-depleted erythrocyte lysate (ECL) and with several actin preparations. ECL was prepared by hypotonic lysis of erythrocytes from a healthy pig. Red blood cells were washed three times with $1 \times$ PBS $(500 \times \mathrm{g}, 15 \mathrm{~min}, \mathrm{RT})$ and resuspended in ice cold lysis buffer $\left(5 \mathrm{mM} \mathrm{Na}_{2} \mathrm{PO}_{4}\right.$, $1 \mathrm{mM}$ EDTA, pH 7.6, Sigma) at a 1:1 (v/v) ratio. After incubation on ice for $5 \mathrm{~min}$, the ghosts (remnants of lysed cells) were pelleted $\left(30,000 \times \mathrm{g}, 15 \mathrm{~min}, 4^{\circ} \mathrm{C}\right)$. This procedure was repeated until the pellet turned white. The white pellet was washed in $1 \times$ PBS, homogenised by ultrasonication on ice and IgG depleted. The concentration was set to $1 \mathrm{mg} / \mathrm{ml}$ with $1 \times \mathrm{PBS}$ and the suspension was stored in $1 \mathrm{ml}$ aliquots at $-80^{\circ} \mathrm{C}$ until further use.

Pig and rabbit muscle actin ( $\alpha$-actin) were obtained from Sigma, Buchs, Switzerland. Porcine cytoskeletal actin ( $\beta$-actin) was not commercially available and was therefore produced recombinantly (see below).

\section{Screening of sera by ELISA}

Sera were obtained in previous studies using a splenectomized pig model for experimental M. suis infections [13]. They were divided into two groups. Group 1 consisted of sera from animals that had been immunised with a recombinant immunogenic surface protein of M. suis (MSG1) [13] prior to splenectomy and M. suis infection. Group 2 sera were from splenectomised, M. suis-infected pigs that had not been immunised [11]. The studies were approved by the government of upper Bavaria under the registration number 211-2531-77/98 and were performed in compliance with animal care legal prescriptions.

The porcine sera were screened for autoreactivity by ELISA as described previously [30]. Briefly, $100 \mathrm{ng}$ of IgG-depleted neg Ag were coated on individual wells of microtiter plates. Sera were diluted 1:100 in $1 \times$ PBS containing $0.05 \%(\mathrm{v} / \mathrm{v})$ Tween 20 (PBST) and tested in duplicate. Sera were drawn prior to $M$. suis infection, after immunisation in group 1 (I), during the first clinical attack (II), between first and second clinical attacks (III), and during the second clinical attack (IV). Sera reactive with neg Ag were further tested for reactivity with ECL, pig and rabbit $\alpha$-actin, and porcine $\beta$-actin. The protein concentration was $100 \mathrm{ng} /$ well. OD values $(\lambda=405 \mathrm{~nm})$ were determined and normalised to a negative control (pool of 10 sera from healthy pigs). Cut-off values were set at three times the standard deviation of the negative controls.
To determine the subtypes of autoreactive antibodies, monoclonal antibodies targeting porcine $\mathrm{IgG}_{1}$ and $\mathrm{IgG}_{2}$ (Prionics, Schlieren, Switzerland) were diluted 1:1000 in PBST. Horse-radish-peroxidase-labelled antibodies against mouse IgG (Sigma) diluted 1:5000 in PBST were used for detection.

\section{Cloning and expression of porcine cytoskeletal actin}

Porcine cytoskeletal actin ( $\beta$-actin) was not commercially available. Using the encoding mRNA sequence (Genbank: AY550069), the gene was de novo synthesised and optimised for E. coli codon usage by Eurofins (Martinsried, Germany). For cloning, specific recognition sites for endonucleases were introduced (XhoI at the 5 ' end and HindIII at the 3' end). For expression, the gene was ligated into the expression vector $\mathrm{pBadMycHisA}$ (Invitrogen) using T4 ligase from Roche, Basel, Switzerland (overnight, $14^{\circ} \mathrm{C}$ ). The insert-containing vector was transformed into E. coli LMG194 (Invitrogen). The E. coli transformants were grown at $37^{\circ} \mathrm{C}$, and expression of the recombinant porcine $\beta$-actin was induced by adding $0.02 \%$ arabinose at $\mathrm{OD}_{600}=0.4$. The cells were incubated for a further $4 \mathrm{~h}$ at $37^{\circ} \mathrm{C}$ and the $6 \times$ His-tagged protein was purified by nickel affinity chromatography (GE Healthcare, Glattbrugg, Switzerland) according to the manufacturer's recommendations. To extract the protein from inclusion bodies, $8 \mathrm{M}$ urea was used for purification. The recombinant protein was further purified by electro-elution from $10 \%$ polyacrylamide gels using an Electro-Eluter Model 422 (Biorad, Reinach, Switzerland). The eluted protein was precipitated by adding three volumes of ice cold acetone and incubating overnight at $-20^{\circ} \mathrm{C}$. The protein was harvested by centrifugation $\left(10,000 \times \mathrm{g}, 90 \mathrm{~min}, 4^{\circ} \mathrm{C}\right)$, allowed to dry, resuspended in $1 \times \mathrm{PBS}$, and stored in aliquots of $1 \mathrm{mg} / \mathrm{ml}$ at $-80^{\circ} \mathrm{C}$.

\section{Production of hyperimmune sera}

One milligram of protein (recombinant MSG1 [13], recombinant porcine $\beta$-actin or porcine $\alpha$-actin (Sigma, Buchs, Switzerland)) was mixed with complete Freund's adjuvant (Sigma) at a 1:1 (v/v) ratio and injected subcutaneously into rabbits. Two booster injections (the proteins were mixed 1:1 (v/v) with incomplete Freund's adjuvant) were given two and four weeks later. Six weeks after the first injection the rabbits were bled; the serum was harvested and stored in aliquots at $-20^{\circ} \mathrm{C}$. Immunisations were approved by the Veterinary Office of Zurich and conducted under the registration number $144 / 2008$ in accordance with legal prescriptions.

\section{Western blotting}

The cross-reactivity of rabbit sera against MSG1 and porcine $\alpha$-actin between $\alpha$-actin and MSG1 was tested by western blotting as described previously [11]. Briefly, 
$\alpha$-actin and MSG1 were loaded on $10 \%$ polyacrylamide gels containing $1 \% \operatorname{SDS}(\mathrm{w} / \mathrm{v})$ and transferred to a nitrocellulose membrane (Whatman Protran', GE Healthcare). Free binding positions on the membrane were blocked with skim milk (Sigma, 2\% w/v in Tris buffered saline, TBS) and probed with rabbit hyperimmune sera (diluted 1:100 in TBS containing $2 \%$ skim milk powder) against recombinant MSG1 and $\alpha$-actin, respectively. A secondary antibody reactive with pig IgG (Sigma) and coupled to horseradish peroxidase (HRP) was used, diluted 1:5000 in TBS containing $2 \%(\mathrm{w} / \mathrm{v})$ skim milk powder. The substrate for the HRP was $\mathrm{H}_{2} \mathrm{O}_{2}$ (Sigma); 4-Chloro-1-naphthol (Sigma) was used as chromogen. The reaction was stopped by adding MQ water (Millipore, Zug, Switzerland).

\section{Search for cross-reactive epitopes}

To identify potential cross-reactive epitopes, an epitope finder program referred to as the NetMHCpan algorithm was used [21]. Published protein sequences of MSG1 (UniProtKB/TrEMBL:Q05G10), porcine $\alpha$-actin (Swiss-Prot:P68137.1) and porcine $\beta$-actin (Swiss-Prot: Q6QAQ1.2) were used as input.

\section{Statistical analysis}

The ELISA OD values measured at $405 \mathrm{~nm}$ were compared by linear regression models using SigmaPlot software, Version 10.0 (SPSS Inc., Chicago, IL, U.S.A). To show independence of values between the two groups, the unpaired Student's $t$-test was applied (significant difference if $P \leq 0.05$ ).

\footnotetext{
Acknowledgements

This work was supported both by the Jubiläumsspende (material costs) and the Forschungskredit (PhD salary of KMF) of the University of Zurich, Switzerland. Anja Hamburger is acknowledged for expert technical help.
}

\section{Author details}

${ }^{1}$ Institute of Veterinary Bacteriology, Vetsuisse Faculty, University of Zurich, Switzerland. ${ }^{2}$ Clinic for Swine, Ludwig-Maximilians-University Munich, Munich, Germany. ${ }^{3}$ Clinic for Swine, University for Veterinary Medicine, Vienna, Austria.

\section{Authors' contributions}

KMF performed the experiments with group 1 sera and drafted the manuscript; $\mathrm{KH}$ performed the experiments with group 2 sera and helped to draft the manuscript; $K$ Heinritzi designed the group 2 animal experiments and performed them; the animal experiments of group 1 were designed and organised by MR; LH designed, planned and coordinated the study overall, supervised the experiments and helped to draft the manuscript. All authors read and approved the final manuscript.

Received: 9 December 2009 Accepted: 30 March 2010 Published: 30 March 2010

\section{References}

1. Hoelzle LE: Haemotrophic mycoplasmas: recent advances in Mycoplasma suis. Vet Microbiol 2008, 130:215-226.

2. Messick JB: Hemotrophic mycoplasmas (hemoplasmas): a review and new insights into pathogenic potential. Vet Clin Pathol 2004, 33:2-13.
3. Yang D, Tai X, Qiu Y, Yun S: Prevalence of Eperythrozoon spp. infection and congenital eperythrozoonosis in humans in Inner Mongolia, China. Epidemiol Infect 2000, 125:421-426.

4. dos Santos AP, dos Santos RP, Biondo AW, Dora JM, Goldani LZ, de Oliveira ST, de Sa Guimaraes AM, Timenetsky J, de Morais HA, Gonzalez FH, Messick JB: Hemoplasma infection in HIV-positive patient, Brazil. Emerg Infect Dis 2008, 14:1922-1924.

5. Packman $\mathrm{CH}$ : Hemolytic anemia due to warm autoantibodies. Blood Rev 2008, 22:17-31.

6. Petz LD: Cold antibody autoimmune hemolytic anemias. Blood Rev 2008, 22:1-15.

7. Herold G: Autoimmunhaemolytische Anämien (AlHA). Innere Medizin Herold G Köln 2006, 43-45.

8. Sokol RJ, Booker DJ, Stamps R: The pathology of autoimmune haemolytic anaemia. J Clin Pathol 1992, 45:1047-1052.

9. Hoffmann R, Schmid DO, Hoffmann-Fezer G: Erythrocyte antibodies in porcine eperythrozoonosis. Vet Immunol Immunopathol 1981, 2:111-119.

10. Zachary JF, Smith AR: Experimental porcine eperythrozoonosis: Tlymphocyte suppression and misdirected immune responses. Am J Vet Res 1985, 46:821-830.

11. Hoelzle LE, Hoelzle K, Ritzmann M, Heinritzi K, Wittenbrink MM: Mycoplasma suis antigens recognized during humoral immune response in experimentally infected pigs. Clin Vaccine Immunol 2006, 13:116-122.

12. Hoelzle LE, Hoelzle K, Harder A, Ritzmann M, Aupperle H, Schoon HA, Heinritzi K, Wittenbrink MM: First identification and functional characterization of an immunogenic protein in unculturable haemotrophic Mycoplasmas (Mycoplasma suis HspA1). FEMS Immunol Med Microbiol 2007, 49:215-223.

13. Hoelzle K, Doser S, Ritzmann M, Heinritzi K, Palzer A, Elicker S, Kramer M, Felder KM, Hoelzle LE: Vaccination with the Mycoplasma suis recombinant adhesion protein MSG1 elicits a strong immune response but fails to induce protection in pigs. Vaccine 2009, 27:5376-5382

14. Schreiber AD, Frank MM: Role of antibody and complement in the immune clearance and destruction of erythrocytes. I. In vivo effects of IgG and IgM complement-fixing sites. J Clin Invest 1972, 51:575-582.

15. Johnson GD, Holborow EJ, Glynn LE: Antibody to smooth muscle in patients with liver disease. Lancet 1965, 2:878-879.

16. Lutz HU: Homeostatic roles of naturally occurring antibodies: an overview. J Autoimmun 2007, 29:287-294.

17. Biberfeld G, Gronowicz E: Mycoplasma pneumoniae is a polyclonal B-cell activator. Nature 1976, 261:238-239.

18. Fossati-Jimack L, Azeredo da Silveira S, Moll T, Kina T, Kuypers FA, Oldenborg PA, Reininger $L$, Izui $S$ : Selective increase of autoimmune epitope expression on aged erythrocytes in mice: implications in antierythrocyte autoimmune responses. J Autoimmun 2002, 18:17-25.

19. Groebel K, Hoelzle K, Wittenbrink MM, Ziegler U, Hoelzle LE: Mycoplasma suis invades porcine erythrocytes. Infect Immun 2009, 77:576-584.

20. Zachary JF, Basgall EJ: Erythrocyte membrane alterations associated with the attachment and replication of Eperythrozoon suis: a light and electron microscopic study. Vet Pathol 1985, 22:164-170.

21. Hoof I, Peters B, Sidney J, Pedersen LE, Sette A, Lund O, Buus S, Nielsen M: NetMHCpan, a method for MHC class I binding prediction beyond humans. Immunogenetics 2009, 61:1-13.

22. Kohm AP, Fuller KG, Miller SD: Mimicking the way to autoimmunity: an evolving theory of sequence and structural homology. Trends Microbiol 2003, 11:101-105.

23. Wucherpfennig KW: Mechanisms for the induction of autoimmunity by infectious agents. J Clin Invest 2001, 108:1097-1104.

24. Fujinami RS, Oldstone MB: Amino acid homology between the encephalitogenic site of myelin basic protein and virus: mechanism for autoimmunity. Science 1985, 230:1043-1045.

25. Fischer JT, Petz LD, Garratty G, Cooper NR: Correlations between quantitative assay of red cell-bound $\mathrm{C} 3$, serologic reactions, and hemolytic anemia. Blood 1974, 44:359-373.

26. Lucisano Valim YM, Lachmann PJ: The effect of antibody isotype and antigenic epitope density on the complement-fixing activity of immune complexes: a systematic study using chimaeric anti-NIP antibodies with human $\mathrm{F}_{\mathrm{c}}$ regions. Clin Exp Immunol 1991, 84:1-8.

27. Schenkein HA, Barbour SE, Tew JG: Cytokines and inflammatory factors regulating immunoglobulin production in aggressive periodontitis. Periodontol 2000 2007, 45:113-127. 
28. Hoelzle LE, Adelt D, Hoelzle K, Heinritzi K, Wittenbrink MM: Development of a diagnostic PCR assay based on novel DNA sequences for the detection of Mycoplasma suis (Eperythrozoon suis) in porcine blood. Vet Microbiol 2003, 93:185-196.

29. Hall SM, Cipriano JA, Schoneweis DA, Smith JE, Fenwick BW: Isolation of infective and non-infective Eperythrozoon suis bodies from the whole blood of infected swine. Vet Rec 1988, 123:651.

30. Hoelzle LE, Hoelzle K, Wittenbrink MM: Expression of the major outer membrane protein (MOMP) of Chlamydophila abortus, Chlamydophila pecorum, and Chlamydia suis in Escherichia coli using an arabinoseinducible plasmid vector. J Vet Med B Infect Dis Vet Public Health 2003, 50:383-389.

doi:10.1186/1746-6148-6-18

Cite this article as: Felder et al: Antibodies to actin in autoimmune haemolytic anaemia. BMC Veterinary Research 2010 6:18.

\section{Submit your next manuscript to BioMed Central} and take full advantage of:

- Convenient online submission

- Thorough peer review

- No space constraints or color figure charges

- Immediate publication on acceptance

- Inclusion in PubMed, CAS, Scopus and Google Scholar

- Research which is freely available for redistribution

Submit your manuscript at www.biomedcentral.com/submit
C Biomed Central 